\title{
Adubação fosfatada no crescimento e nos teores de nutrientes em cladódios de pitaia vermelha
}

\section{Phosphorus fertilization on growth and levels of nutrients in red pitaya cladodes}

\author{
Rodrigo Amato Moreira \\ Instituto Federal do Norte de Minas Gerais, Campus Januária \\ E-mail: rodrigo.moreira@ifnmg.edu.br \\ OrclD: 0000-0001-8896-6958
}

\begin{abstract}
Maria do Céu Monteiro da Cruz
Universidade Federal dos Vales do Jequitinhonha e Mucuri, Campus Diamantina

E-mail: mariceu@ufvjm.edu.br

OrclD: 0000-0003-1293-1569
\end{abstract}

Denison Ramalho Fernandes

Universidade Federal dos Vales do Jequitinhonha e Mucuri, Campus Diamantina

E-mail: denison ramalho@yahoo.com.br

OrclD: 0000-0001-7950-5180

Jéssica de Oliveira

Universidade Federal dos Vales do Jequitinhonha e Mucuri, Campus Diamantina

E-mail: jeoliveira agr@hotmail.com

OrclD: 0000-0003-0444-5288

Resumo: Esta pesquisa foi realizada com o objetivo de avaliar o crescimento e a nutrição da pitaia vermelha (Hylocereus undatus) em função da adubação fosfatada. $O$ experimento foi realizado em casa de vegetação em Diamantina, Minas Gerais, Brasil. Estacas de pitaia vermelha (0,20 m de comprimento) foram plantadas em vasos plásticos com capacidade de $4 \mathrm{dm}^{-3}$ de solo. O experimento foi conduzido em delineamento de blocos casualizados, com cinco doses de $\mathrm{P}_{2} \mathrm{O}_{5}\left(0,45,90,180\right.$ e $360 \mathrm{mg}$ por dm ${ }^{-3}$ de solo) incorporadas ao solo, com quatro repetições, três vasos plásticos por parcela e uma estaca por vaso. Dez meses após o plantio, a massa seca dos cladódios e as raízes foram avaliadas. Os cladódios foram levados para o laboratório para análise química de nutrientes. A aplicação de $180 \mathrm{mg}$ de $\mathrm{P}_{2} \mathrm{O}_{5}$ por $\mathrm{dm}^{3}$ de solo promoveu o maior crescimento e proporcionou níveis adequados de nutrientes nos cladódios da pitaia vermelha.

Palavras-chave: fruta do dragão, Hylocereus undatus, pitahaya, superfosfato

\begin{abstract}
This research was conducted to evaluate the growth and nutrition of the red pitaya (Hylocereus undatus) as function of phosphorus fertilization. The experiment was performed in a greenhouse at Diamantina, Minas Gerais, Brazil. Red pitaya cuttings $\left(0,20 \mathrm{~m}\right.$ long) were planted in plastic pots with a $4 \mathrm{dm}^{-3}$ soil capacity. The experiment was conducted in completely randomized block design, with five doses of $\mathrm{P}_{2} \mathrm{O}_{5}(0,45,90,180$ and $360 \mathrm{mg} \mathrm{per} \mathrm{dm}^{-3}$ of soil) incorporated into the soil, with four replications, three plastic pots by plot and one cutting per pot. Ten months after planting, the dry mass of the cladodes and roots were evaluated. Cladodes were taken to the laboratory for chemical analysis of nutrients. The application of $180 \mathrm{mg}$ of $\mathrm{P}_{2} \mathrm{O}_{5}$ per $\mathrm{dm}^{3}$ of soil promoted the greatest growth and provided adequate levels of nutrients in cladodes of the red pitaya.
\end{abstract}

Keywords: dragon fruit, Hylocereus undatus, pitahaya, superphosphate 
Data de recebimento: $14 / 12 / 2018$

Data de aprovação: 12/07/2019

DOI: https://doi.org/10.30612/agrarian.v13i49.9148

\section{Introdução}

A fruta do dragão ou pitaia é uma planta cactácea, que possui um caule triangular fotossinteticamente ativo, conhecido como cladódio. Esta planta tem alto potencial para uso como planta ornamental, frutífera e industrial como fonte de compostos com alta demanda nos mercados nacional e internacional (Ortiz-Hernández \& Carrillo-Salazar, 2012).

No Brasil, a pitaia comercializada no CEAGESP, principal canal de comercialização do país, apresentou aumento de 81,5 toneladas em 2012 para 604 toneladas em 2017 (Ceagesp, 2019) , com os meses de maior oferta de dezembro para abril (Watanabe \& Oliveira, 2014), período reprodutivo para a agricultura no Sudeste do Brasil (Marques et al., 2011; Silva et al., 2015).

O interesse mundial nessa nova fruta é notável, com o número de publicações relacionadas à sua cultura crescendo rapidamente, especialmente durante a última década (Mizrahi, 2014). No entanto, estudos experimentais no Brasil sobre a fertilização e nutrição da pitaia ainda são escassos (Almeida et al., 2016). Algumas publicações têm relatado crescimento e produção com adubo orgânico (Cavalcanti et al., 2011; Moreira et al., 2011, 2012), e outras sobre o crescimento inicial e produção de mudas de pitaia vermelha com adubação química (Almeida et al. , 2014; Corrêa et al., 2014).

Nesse sentido, pesquisas são necessárias para estudar o suprimento de nutrientes à pitaia, principalmente o fósforo, por ser um elemento essencial para o crescimento, desenvolvimento e produção de plantas. Além disso, esse nutriente está associado à fotossíntese, divisão celular e uso de açúcar e amido (López-Bucio et al., 2002). Ainda, segundo esses autores, a deficiência de fósforo pode reduzir o desenvolvimento das plantas porque o fósforo é necessário para estimular o crescimento das raízes.

Para a adequada aplicação do fósforo, o conhecimento da dinâmica dos nutrientes e suas interações com o solo é necessário, pois o fósforo é pouco móvel no solo, e a absorção pelas raízes ocorre principalmente pela difusão, que depende da umidade do solo e da superfície radicular (Gahoonia et al., 1994).

A determinação do teor de fósforo disponível no solo e os níveis de nutrientes nos cladódios da pitaia vermelha também são importantes para determinar a fertilização balanceada que promoverá melhor rendimento agrícola.

Assim, este estudo foi realizado com o objetivo de avaliar o crescimento e a nutrição da pitaia vermelha em função da adubação fosfatada.

\section{Material e Métodos}

A pesquisa foi realizada em casa de vegetação na Universidade Federal dos Vales do Jequitinhonha e

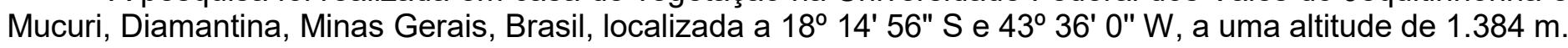
O clima é classificado como temperado úmido, com inverno seco e verão chuvoso, temperatura média anual de $18,3^{\circ} \mathrm{C}$ e precipitação de aproximadamente $1.400 \mathrm{~mm}$ (Vieira et al., 2010). O experimento foi conduzido no período de abril de 2013 a fevereiro de 2014 e, durante esse período, a temperatura média na casa de vegetação foi de $25^{\circ} \mathrm{C} \pm 7^{\circ} \mathrm{C}$.

Foram utilizadas estacas de pitaia vermelha saudáveis [Hylocereus undatus (Haw.) Britton \& Rose], com $0,20 \mathrm{~m}$ de comprimento. As estacas foram seccionadas para remover a dominância apical e antes do plantio passaram por um período de cicatrização de três dias à sombra.

As estacas foram plantadas em vasos plásticos com capacidade de $4 \mathrm{dm}^{3}$ de solo. Os vasos plásticos foram preenchidos com LATOSSOLO VERMELHO-AMARELO Distrófico (Santos et al. 2013), contendo 63\% de areia, $23 \%$ de argila, $14 \%$ de silte e com as seguintes características: $\mathrm{pH}=5,10, \mathrm{P}=1,83 \mathrm{mg} \mathrm{dm}^{-3}, \mathrm{~K}=22,80$ $\mathrm{mg} \mathrm{dm}{ }^{-3}, \mathrm{Ca}=1,00 \mathrm{cmolc} \mathrm{dm}^{-3}, \mathrm{Mg}=0,30 \mathrm{cmol}_{\mathrm{c}} \mathrm{dm}^{-3}, \mathrm{Al}=0,50 \mathrm{cmol}_{\mathrm{c}} \mathrm{dm}^{-3}$, matéria orgânica $=0,40 \mathrm{dag} \mathrm{kg}^{-1}$, saturação por bases $(V)=28 \%$ e capacidade de troca catiônica potencial $(T)=5,10 \mathrm{cmol}_{\mathrm{c}} \mathrm{dm}^{-3}$. Os extratores 
foram: $\mathrm{P}$ e $\mathrm{K}=$ Mehlich-1; $\mathrm{Ca}, \mathrm{Mg}$ e $\mathrm{Al}=\mathrm{KCl} 1 \mathrm{~mol} \mathrm{~L}^{-1}$ e $\mathrm{H}+\mathrm{Al}=$ acetato de cálcio. As análises de solo foram realizadas de acordo com Teixeira et al. (2017).

Um grama de calcário dolomítco (PRNT $=87,0 \%$ ) foi adicionado por $\mathrm{dm}^{3}$ de solo para aumentar a saturação de base a $60 \%$. Posteriormente, o solo foi irrigado e coberto com sacos de polietileno por 15 dias antes das estacas serem plantadas.

No plantio, $25 \mathrm{mg}$ de FTE BR12 por $\mathrm{dm}^{3}$ de solo foram aplicados. Este fertilizante continha Ca $(7,1 \%)$, $\mathrm{S}(5,7 \%), \mathrm{B}(1,8 \%), \mathrm{Cu}(0,8 \%), \mathrm{Mn}(2,0 \%)$, Mo $(0,1 \%)$ e $\mathrm{Zn}(9,0 \%)$. Adicionalmente, foram aplicadas cinco doses de $\mathrm{P}_{2} \mathrm{O}_{5}\left(0,45,90,180\right.$ e $360 \mathrm{mg}$ por $\mathrm{dm}^{3}$ de solo) incorporadas ao solo, em delineamento de blocos casualizados, com quatro repetições, três vasos de plástico por parcela e uma estaca por vaso. O superfosfato simples foi usado como fonte de fósforo ( $18 \% \mathrm{P}_{2} \mathrm{O}_{5}, 16 \%$ Ca e $\left.8 \% \mathrm{~S}\right)$.

As plantas foram fertilizadas com sulfato de amônio e como fontes de $\mathrm{N}$ para fornecer as mesmas quantidades de $\mathrm{S}$, e cloreto de potássio serviu como fonte de $\mathrm{K}$. Para isso, $300 \mathrm{mg}$ de $\mathrm{N}$ por $\mathrm{dm}^{3}$ de solo e 150 $\mathrm{mg}$ de $\mathrm{K}$ por $\mathrm{dm}^{3}$ de solo foram aplicados, dividido em três aplicações entre os meses de junho, setembro e dezembro de 2013.

A irrigação foi realizada diariamente por nebulização, mantendo o solo próximo da capacidade de campo. Estacas de madeira ( $1 \mathrm{~m}$ de comprimento) e tiras de polietileno foram utilizados em cada vaso para tutorar as pitaias. Todas as brotações (cladódios) foram apoiadas sem realizar a poda.

Aos dez meses após o plantio, as plantas foram segmentadas e separadas em cladódios e raízes. Todas as partes foram lavadas em água e levadas à estufa de circulação de ar forçada por 120 horas para secagem a uma temperatura de $65^{\circ} \mathrm{C}$ para posterior determinação da massa seca ( $\mathrm{g}$ ) de cada segmentação.

Os cladódios foram levados ao laboratório para análise química dos nutrientes, realizada de acordo com o método proposto por Malavolta et al. (1997). O teor total de $\mathrm{N}$ foi determinado pelo método de Kjeldahl após digestão com ácido sulfúrico; Os teores de $\mathrm{P}, \mathrm{K}$ e S foram determinados após digestão nitro-perclórica, sendo o teor de $\mathrm{P}$ determinado para os extratos analisados por colorimetria, o teor de $\mathrm{K}$ determinado pela fotometria de chama e o S por espectrofotometria. Os teores de Ca e Mg foram determinados por espectrofotometria de absorção atômica.

$\mathrm{O}$ solo de cada parcela no final do experimento foi enviado para análise química. $\mathrm{P}$ e $\mathrm{K}$ foram extraídos por Mehlich-1; Ca, Mg e Al ${ }^{3+}$ foram extraídos com KCl $1 \mathrm{~mol} \mathrm{~L}^{-1} \mathrm{e} \mathrm{H}+$ Al por acetato de cálcio.

Os dados foram submetidos à análise de variância e análise de regressão polinomial $(p<0,05)$.

\section{Resultados}

A aplicação de fósforo influenciou de maneira quadrática o crescimento de cladódios e de raízes da pitaia vermelha (Figura 1). A adição de $180 \mathrm{mg}$ de $\mathrm{P}_{2} \mathrm{O}_{5}$ por $\mathrm{dm}^{3}$ de solo promoveu a máxima massa seca com aumento de $205,8 \%$ no crescimento de cladódios, e a dose estimada de $175 \mathrm{mg}$ de $\mathrm{P}_{2} \mathrm{O}_{5}$ por $\mathrm{dm}^{3}$ de solo proporcionou a máxima massa seca com $136,0 \%$ de crescimento nas raízes da pitaia vermelha, em comparação com plantas sem a aplicação de fósforo (Figuras 1A e 1B). 
(A)

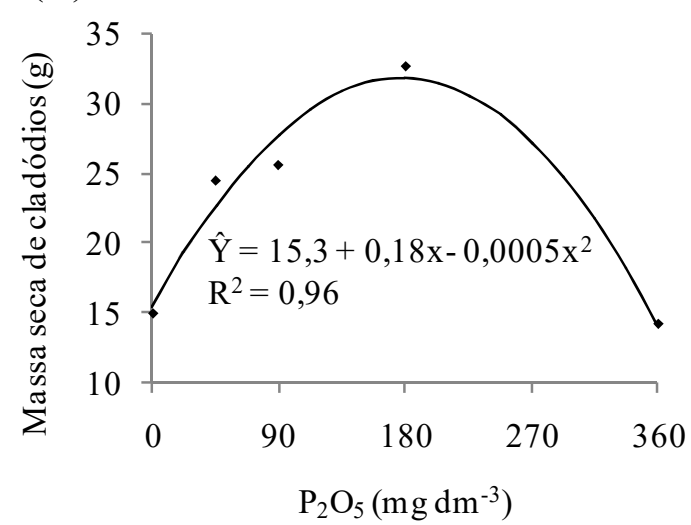

(B)

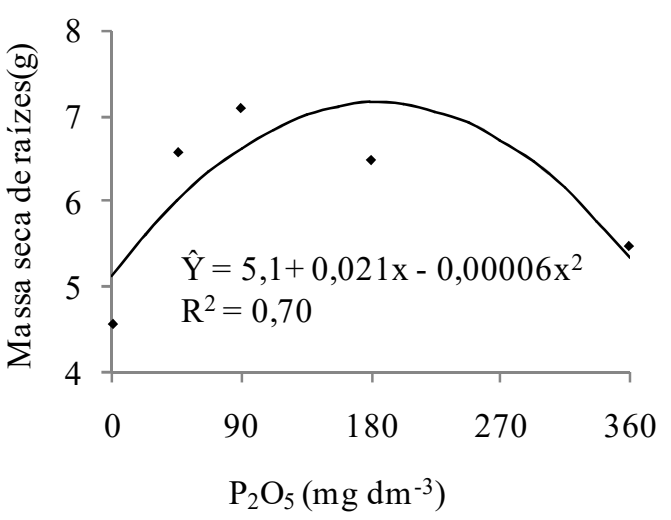

Figura 1. (A) Massa seca de cladódios (g) e (B) massa seca de raízes (g) por planta de pitaia vermelha em função da aplicação de $\mathrm{P}_{2} \mathrm{O}_{5}\left(\mathrm{mg} \mathrm{dm}^{-3}\right)$.

A elevação das doses de $\mathrm{P}_{2} \mathrm{O}_{5}$ promoveu aumento linear na disponibilidade de $\mathrm{P}$ no solo (Figura 2). No entanto, a aplicação de fósforo não afetou o restante da composição química do solo, que foi avaliada no final do experimento (Tabela 1).

Os teores de $\mathrm{N}, \mathrm{K}$ e P nos cladódios de pitaia vermelha aumentaram linearmente com doses de $\mathrm{P}_{2} \mathrm{O}_{5}$ (Figura 3A). O maior teor de cálcio $\left(17,86 \mathrm{~g} \mathrm{~kg}^{-1}\right)$ nos cladódios foi observado com a aplicação de 156,6 mg de $\mathrm{P}_{2} \mathrm{O}_{5}$ por $\mathrm{dm}^{3}$ de solo. Os teores de $\mathrm{Mg}$ e $\mathrm{S}$ nos cladódios não foram afetados pela aplicação de fósforo no solo (Figura 3B).

Os teores de Zn, Fe, Cu (Figura 4A) e B (Figura 4B) em cladódios de pitaia vermelha permaneceram semelhantes com diferentes adubações fosfatadas; no entanto, o Mn diminuiu linearmente com a crescente aplicação do $\mathrm{P}_{2} \mathrm{O}_{5}$.

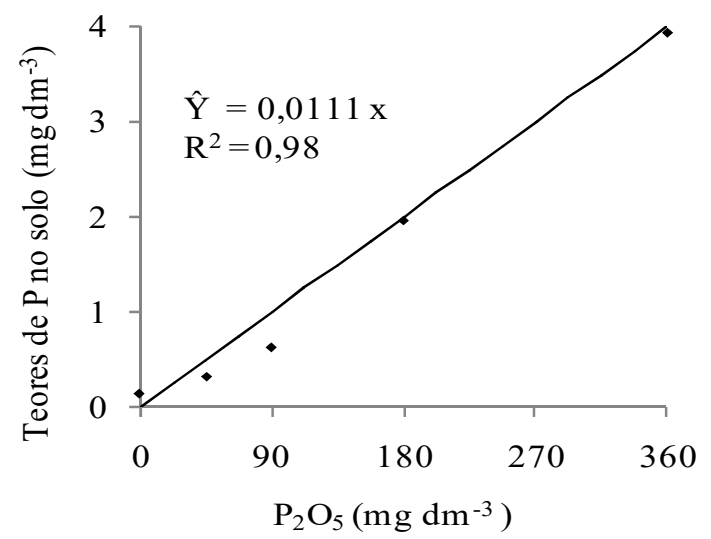

Figura 2. Teores de $\mathrm{P}$ no solo $\left(\mathrm{mg} \mathrm{dm}^{-3}\right)$ em função da aplicação de $\mathrm{P}_{2} \mathrm{O}_{5}\left(\mathrm{mg} \mathrm{dm}^{-3}\right)$ no final do experimento. 


\section{orgarian}

Tabela 1. Análise química do solo no final do experimento.

\begin{tabular}{ccccccccc}
$\mathrm{P}_{2} \mathrm{O}_{5}$ & $\mathrm{~K}$ & $\mathrm{pH}$ & $\mathrm{Ca}$ & $\mathrm{Mg}$ & $\begin{array}{c}\mathrm{Al} \\
\mathrm{H}\end{array}$ & $\mathrm{H}+\mathrm{Al}$ & $\mathrm{CTC}$ & $\begin{array}{c}\mathrm{V} \\
----(\%)--\end{array}$ \\
\hline 0 & 50,0 & 5,1 & 0,69 & 0,60 & 0,11 & 2,7 & 1,52 & 34,5 \\
45 & 46,3 & 5,6 & 0,67 & 0,50 & 0,06 & 2,2 & 1,33 & 37,5 \\
90 & 23,5 & 5,5 & 0,68 & 0,49 & 0,06 & 2,5 & 1,29 & 33,0 \\
180 & 35,0 & 5,0 & 0,72 & 0,66 & 0,12 & 3,0 & 1,59 & 33,0 \\
360 & 27,5 & 5,1 & 0,71 & 0,65 & 0,17 & 3,6 & 1,59 & 28,5 \\
\hline $\mathrm{CV}(\%)$ & 27,8 & 4,9 & 9,8 & 15,2 & 22,4 & 16,5 & 8,2 & 9,5 \\
\hline
\end{tabular}

Médias não significativas $(p<0,05)$. CTC: Capacidade de troca de cátions $\left(\mathrm{CTC}=\sum \mathrm{K}, \mathrm{Ca}, \mathrm{Mg}, \mathrm{H}+\mathrm{Al}\right)$. V: Saturação de bases $=\left(\sum \mathrm{Ca}\right.$, $\mathrm{Mg}, \mathrm{K}) /(\mathrm{CTC}) \times 100$.
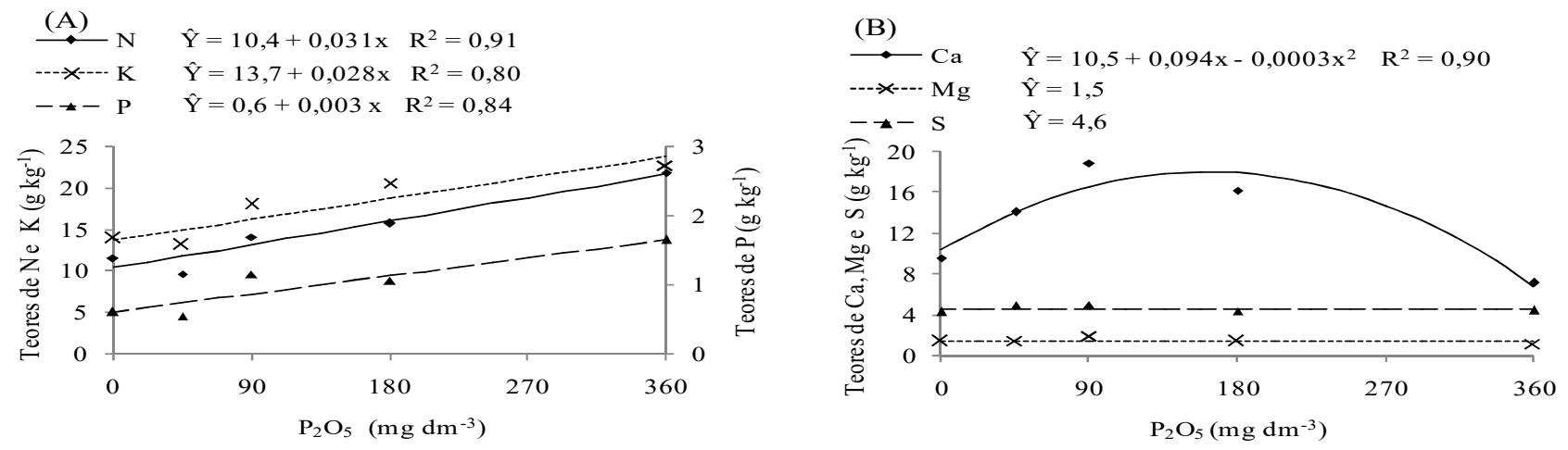

Figura 3. Teores de macronutrientes $\left(\mathrm{g} \mathrm{kg}^{-1}\right)$ em cladódios de pitaia vermelha: $(A) \mathrm{N}, \mathrm{K}$ e $\mathrm{P}$ e (B) $\mathrm{Ca}, \mathrm{Mg}$ e $\mathrm{S}$ em função da aplicação de $\mathrm{P}_{2} \mathrm{O}_{5}\left(\mathrm{mg} \mathrm{dm}^{-3}\right)$.
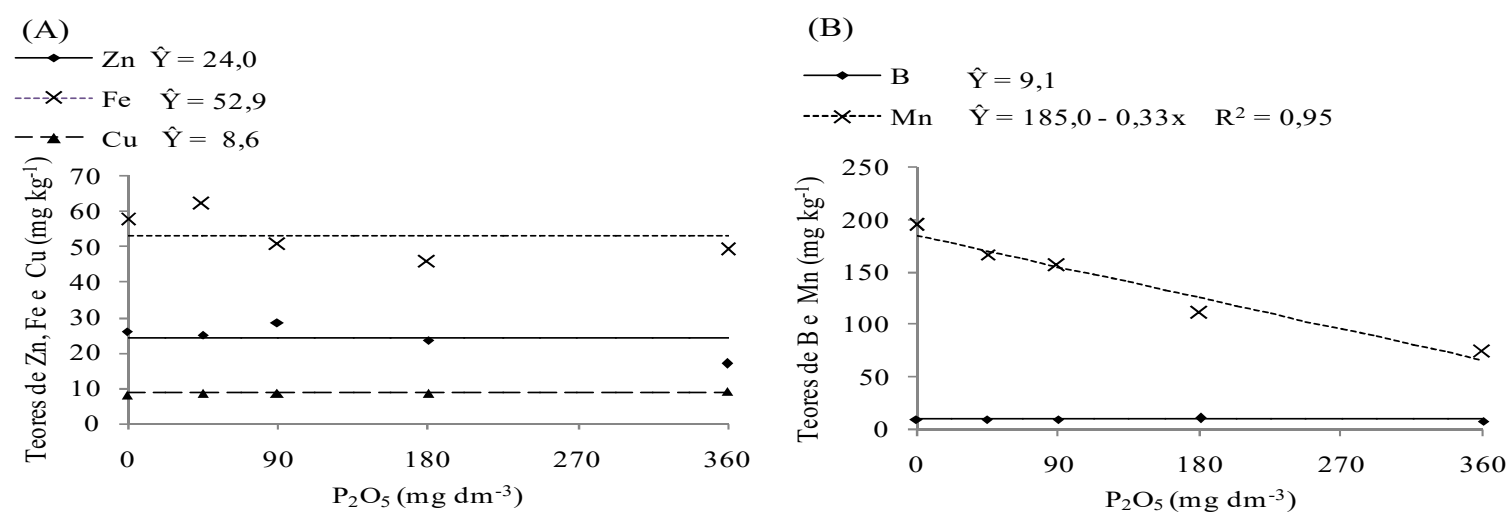

Figura 4. Teores de micronutrientes $\left(\mathrm{mg} \mathrm{kg}^{-1}\right)$ em cladódios de pitaia vermelha (A) Zn, Fe e Cu e (B) B e Mn em função da aplicação de $\mathrm{P}_{2} \mathrm{O}_{5}\left(\mathrm{mg} \mathrm{dm}^{-3}\right)$.

\section{Discussão}

A aplicação de fósforo para o crescimento inicial das plantas é indicada pelas funções desse nutriente na fotossíntese e no início do crescimento nas raízes, o que melhora a eficiência do uso da água, absorção e 
utilização de outros nutrientes (Malavolta et al., 1997). Além disso, o fósforo requer mais atenção devido aos baixos níveis observados nos solos tropicais (Prado et al., 2005).

No entanto, a aplicação deve ser feita em quantidade adequada que promova boa disponibilidade de nutrientes para as plantas. No presente experimento, a aplicação de $180 \mathrm{mg}$ de $\mathrm{P}_{2} \mathrm{O}_{5}$ por $\mathrm{dm}^{3}$ de solo elevou o teor no solo para cerca de $2 \mathrm{mg}$ de $\mathrm{P}$ por $\mathrm{dm}^{3}$ (Figura 2) aos dez meses após o plantio. Assim, essa aplicação promoveu o maior crescimento dos cladódios e do sistema radicular da pitaia vermelha. Isso porque o fósforo favorece o desenvolvimento da planta e estimula a produção de biomassa (Dias et al., 2009).

A rápida formação e crescimento das raízes da pitaia ocorreu a partir do suprimento de fósforo no solo, porque a disponibilidade adequada desse nutriente para a planta altera as condições deficientes. Esse comportamento também foi relatado na produção de bananeira 'Prata Anã' no viveiro, em função da adubação fosfatada por Silva et al. (2011).

$\mathrm{O}$ aumento linear dos teores de $\mathrm{P}$ nos cladódios da pitaia vermelha pela aplicação de $\mathrm{P}_{2} \mathrm{O}_{5}$ ao solo (Figura 3A) é atribuído à maior disponibilidade desse nutriente no solo (Figura 2).

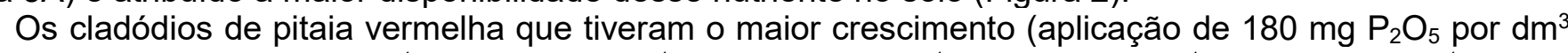
de solo) apresentaram 16,0 g kg-1 de N, 1,13 $\mathrm{g} \mathrm{kg}^{-1}$ de $\mathrm{P}, 18,71 \mathrm{~g} \mathrm{~kg}^{-1}$ de $\mathrm{K}, 18,0 \mathrm{~g} \mathrm{~kg}^{-1} \mathrm{de} \mathrm{Ca}, 1,5 \mathrm{~g} \mathrm{~kg}^{-1} \mathrm{de} \mathrm{Mg}$,

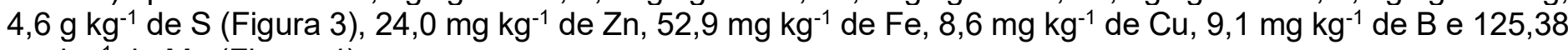
$\mathrm{mg} \mathrm{kg}^{-1}$ de Mn (Figura 4).

Nenhuma quantidade adequada de nutrientes tem sido recomendada para o cultivo de pitaia na literatura. No entanto, os níveis avaliados nesta pesquisa estão próximos aos verificados por Moreira et al. (2012) para o crescimento inicial de pitaia vermelha aos três meses após o plantio com adubação orgânica e granulado bioclástico no Brasil.

A aplicação acima de $180 \mathrm{mg} \mathrm{P}_{2} \mathrm{O}_{5}$ por $\mathrm{dm}^{3}$ de solo promoveu desequilíbrio entre os nutrientes, pois foi observada redução no crescimento dos cladódios e raízes da pitaia vermelha (Figuras $2 \mathrm{~A}$ e 2B).

Os resultados mais satisfatórios neste experimento foram obtidos com menores valores de $\mathrm{P}$ no solo (2 $\mathrm{mg} \mathrm{dm}{ }^{-3}$ ) e no cladódio da pitaia vermelha $\left(1,13 \mathrm{~g} \mathrm{~kg}^{-1}\right)$ quando comparados aos resultados de Corrêa et al. (2014), que relataram valores de 60 a $75 \mathrm{mg}$ de $P$ por dm no substrato e 4,5 a 6,0 g kg-1 de $P$ nos cladódios de pitaia vermelha. Essa diferença pode ser atribuída ao período em que o experimento foi realizado, pois, neste estudo, as plantas e o solo foram avaliados dez meses após o plantio, e esses autores avaliaram as plantas e o solo apenas seis meses após o plantio. Assim, as plantas do presente estudo tiveram mais quatro meses para crescimento e absorção de nutrientes do solo, resultando em diluição nos níveis de P no cladódio e redução no solo.

Os teores de nutrientes observados em cladódios de pitaia vermelha considerados satisfatórios nesta pesquisa de $\mathrm{N}\left(16,00 \mathrm{~g} \mathrm{~kg}^{-1}\right)$ e K $\left(18,71 \mathrm{~g} \mathrm{~kg}^{-1}\right)$ foram inferiores aos valores relatados por Almeida et al. (2014), 20 a $25 \mathrm{~g} \mathrm{~kg}^{-1}$ de $\mathrm{N}$ com 30 a $40 \mathrm{~g} \mathrm{~kg}^{-1}$ de K . Isso pode ser explicado pela maior aplicação desses dois nutrientes e a avaliação ocorrer em um período menor por esses autores.

\section{Conclusão}

A aplicação de $180 \mathrm{mg}$ de $\mathrm{P}_{2} \mathrm{O}_{5}$ por $\mathrm{dm}^{3}$ de solo promoveu o maior crescimento e teores adequados de nutrientes nos cladódios de pitaia vermelha.

\section{Agradecimentos}

Os autores agradecem à Fundação de Amparo à Pesquisa do Estado de Minas Gerais (FAPEMIG) pelo apoio financeiro e à Coordenação de Aperfeiçoamento de Pessoal de Nível Superior (CAPES) pela concessão de bolsas de estudo.

\section{Referências}




\section{(iid)ARARI}

ALMEIDA, E. I. B.; DEUS, J. A. L.; CORREAA, M. C. M.; CRISOSTOMO, L. A.; NEVES, J. C. L. Linha de fronteira e chance matemática na determinação do estado nutricional de pitaia. Revista Ciência Agronômica, v. 47, n. 4, p. 744-754, 2016.

ALMEIDA, E. I. B.; CORREAA, M. C. M.; CRISOSTOMO, L. A.; ARAÚJO, N. A.; SILVA, J. C. V. Nitrogênio e potássio no crescimento de mudas de pitaia [Hylocereus undatus (Haw.) Britton\& Rose]. Revista Brasileira de Fruticultura, v.36, n. 4, p. 1018-1027, 2014.

CAVALCANTE, I. H. L.; MARTINS, A. B. G.; SILVA JÚNIOR, G. B.; ROCHA, L. F.; FALCÃO NETO, R.; CAVALCANTE, L. F. Adubação orgânica e intensidade luminosa no crescimento e desenvolvimento inicial da pitaya em Bom Jesus-PI. Revista Brasileira de Fruticultura, v. 33, n. 3, p. 970-983, 2011.

CEAGESP. Companhia de Entrepostos e Armazéns Gerais de São Paulo. Guia Ceagesp. Disponível em <http://www.ceagesp.gov.br/guia-ceagesp/pitaia/>. Acesso em 27 de fevereiro de 2019.

CORREAA, M. C. M.; ALMEIDA, E. I. B.; MARQUES, V. B.; SILVA, J. C. V.; AQUINO, B. F. Crescimento inicial de pitaia em função de combinações de doses de fósforo-zinco. Revista Brasileira de Fruticultura, v. 36 , n. 1, p. 261-270, 2014.

DIAS, T. J.; PEREIRA, W. E., CAVALCANTE, L. F.; RAPOSO, R. W. C.; FREIRE, J. L. O. Desenvolvimento e qualidade nutricional de mudas de mangabeiras cultivadas em substratos contendo fibra de coco e adubação fosfatada. Revista Brasileira de Fruticultura, v. 31, n. 2, p.512-523, 2009.

GAHOONIA, T. S.; RAZA S.; NIELSEN, N. E. Phosphorus depletion in the rhizosfere as influenced by soil moisture. Plant Soil, v.159, n. 2, p. 213-218, 1994.

LÓPEZ-BUCIO, J. L.; HERNANDEZ-ABREU, E.; SÁNCHEZ-CALDERÓN, L.; NIETOJACOBO, M. F.; SIMPSON, J.; HERRERA-ESTRELLA, L. Phosphate availability alters architecture and cause changes in hormone sensitivity in the Arabidopsis root system. Plant Physiology, v.129, p. 244-256, 2002.

MALAVOLTA, E.; VITTI, G. C.; OLIVEIRA, S. A. Avaliação do estado nutricional das plantas: princípios e aplicações. 2. ed. Piracicaba: POTAFOS. 1997, 319 p.

MARQUES, V. B.; MOREIRA, R. A.; RAMOS, J. D.; ARAÚJO, N. A.; SILVA, F. O. R. Fenologia reprodutiva de pitaia vermelha no município de Lavras, MG. Ciência Rural, v. 41, n. 6, p. 984-987, 2011.

MOREIRA, R. A.; RAMOS, J. D.; ARAÚJO, N. A.; MARQUES, V. B. Produção e qualidade de frutos de pitaiavermelha com adubação orgânica e granulado bioclástico. Revista Brasileira de Fruticultura, v. 33, n. esp., p. 762-766, 2011.

MOREIRA, R. A.; RAMOS, J. D.; ARAÚJO, N. A.; MARQUES, V. B.; MELO, P. C. Produtividade e teores de nutrientes em cladódios de pitaia vermelha utilizando-se adubação orgânica e granulado bioclástico. Revista Brasileira de Ciências Agrárias, v.7, suppl., p.714-719, 2012.

MIZRAHI, Y. Vine-cacti pitayas - the new crops of the world. Revista Brasileira de Fruticultura, v. 36, n.1, p.124-138, 2014.

ORTIZ-HERNÁNDEZ, Y. D.; CARRILLO-SALAZAR, J. A. Pitahaya (Hylocereus spp.): a short review. Comunicata Scientiae, v.3, n. 4, p. 220-237, 2012.

PRADO, R. M.; VALE, D.W.; ROMUALDO, L. M. Fósforo na nutrição e produção de mudas de maracujazeiro. Acta Scientiarum. Agronomy, v. 27, n. 3, p. 493-498, 2005.

SANTOS, H. G.; JACOMINE, P. K. T.; ANJOS, L. H. C.; OLIVEIRA, V. A.; LUMBRERAS, J. F.; COELHO, M. R.; ALMEIDA, J. A.; CUNHA, T. J. F.; OLIVEIRA, J. B. Sistema Brasileiro de Classificação de Solos. 3.ed. rev. e ampl. Brasília: Embrapa. 2013, 353 p.

SILVA, J. T. A.; SILVA, I. P.; PEREIRA, R. D. Adubação fosfatada em mudas de bananeira 'Prata anã'(AAB), cultivadas em dois Latossolos. Revista Ceres, v. 58, n. 2, p. 238-242, 2011.

Agrarian, Dourados, v. 13, n. 49, p. 377-384, 2020.

Este obra está licenciada com uma Licença Creative Commons Atribuição-NãoComercial-CompartilhaIqual 3.0 Brasil. 
